\title{
Smart Cognitive Cellular Network
}

\author{
Saima Khadim ${ }^{1}$, Adnan Waqar ${ }^{1}$, Aamir Zeb ${ }^{2}$, Imran Khan ${ }^{1}$ and Izhar Hussain ${ }^{1}$ \\ ${ }^{1}$ Dawood University of Engineering and Technology, Karachi, Pakistan \\ ${ }^{2}$ NED University of Engineering and Technology, Karachi, Pakistan
}

\begin{abstract}
The number of wireless devices are increasing rapidly with the advent of technological innovation. Many of these devices require large bandwidth, hence as a consequence spectrum scarcity is also increasing rapidly. Cognitive radio is a promising technology that can provide solution to spectrum scarcity by dynamic frequency allocation. So far, frequency allocation has been static. Dynamic spectrum allocation allows secondary users to use frequencies assigned to primary (licensed) users without causing interference to primary users. In this paper, we have developed a dataset utilization of different frequencies by spectrum sensing. This data is used as an input to machine learning algorithm in order to predict white spaces and transmission levels at which secondary users can transmit without creating any interference with primary users. For creation of the database we have performed spectrum sensing on different frequency bands. After analysing the data, we found out spectrum holes (white spaces, where primary user isn't transmitting) and transmission levels of primary user so that secondary user can transmit without creating interference. From this dataset, we trained our machine learning algorithm to accurately predict spectrum holes and transmission levels for secondary users. Using machine learning we were able to predict accurately within a fraction of time. Our proposed methodology increases accuracy and at the same time reduces interference, power consumption and frequency allocation time and then created dataset is import to the Artificial Neural Network (ANN) for the prediction of best available spectrum slots.
\end{abstract}

\section{Introduction}

According to Communication regularity bodies it has been found that a large amount of licensed Spectrum band is not utilized fully, for this unused spectrum to be utilized completely, we use the Cognitive Radio Technology. Cognitive radio is a widely used promising solution of Spectrum Scarcity. The concept of Cognitive radio was first proposed by Joseph Mitola in 1999 as a solution to optimize the unused licensed Spectrum [1]. Cognitive radio system has the ability to sense the unused spectrum by adjusting their parameters in its environment. A basic Cognitive Cycle comprises of three steps

\section{Spectrum Sensing}

2. Spectrum Analysis

3. Spectrum Decision making

The Cognitive Cycle is introduced by Haykin is presented in [2] in Cognitive Radio. The Cognitive Radio Network has two types of user's, Primary user which is also known as licensed user which has the highest priority over the specific spectrum, while Secondary user which is unlicensed user has the lowest priority over the spectrum. In CRN allotted channels are logically divided into different slots, the secondary user senses the activity of the primary user within each slot for certain duration when it is sensed idle

Received (June 15, 2017), Review Result (September 23, 2017), Accepted (October 5, 2017) 
or find spectrum hole then secondary user can accesses the entire spectrum slot without affecting (interference) the licensed user

To mitigate problem of interference to Licenced (Primary) user, Cognitive users require some techniques. There are several techniques for Spectrum Sensing from which some techniques are able to sense the full band of Cellular Network. While due to hardware limitations secondary users only sense one part of the spectrum in a slot. On the additional side sensing energy can be conserved by avoiding the used portion of the spectrum. To make the secondary users efficient to manage the spectrum, spectrum prediction is used. The secondary users can predict the spectrum which is based on the sensing history and sense only if a spectrum slot is predicted to be an idle in the next time slot, so secondary user can save the energy by spectrum sensing. By Spectrum Prediction, predicted spectrum slots can be used by the secondary users without causing any interference and QoS will be increased.

In this paper, dataset of Cellular band (e.g. $850 \& 900 \mathrm{MHz}, 1800 \mathrm{MHz}$ and $2100 \mathrm{MHz}$ ) is created by spectrum sensing, the created dataset is sensed several times and then from this database Artificial neural network is trained and the best available spectrum slots are achieved.

First air interface standard, WRANs (Wireless regional area networks) is being used in some countries. It uses TVWS (TV White Spaces) and hence it's not a complete dynamic method of frequency reuse.

The rest of paper is organized as follow. In Section II we present a Literature Review. In Section III methodology and in Section IV conclusion.

\section{Literature Review}

In [3], the author used two techniques Multilayer Perceptron and Hidden Markov Model for Spectrum prediction. The benefit of channel predictor is that, it does not require any prior knowledge of the channel and also author compared the accuracy of Multilayer Perceptron (MLP) \& Hidden Markov model (HMM). In [4], the motive of this paper is to increase decision accuracy of the best available unused spectrum slots below the various different noisy conditions which is implemented by using Multilayer Perceptron Neural Network. It upgrades the required throughput, by using three types of Neural Network through Simulation which are Perceptron Neural Network, Feed forward Neural Network and Elman Neural Network the performance is analysed and from the simulation results the author concluded that performance of Perceptron Neural Network is slightly better than Feed Forward Neural Network and Elman Neural Network.

In [5] compared the performance of two most popular neural network algorithms, Multilayer Perceptron Neural Network and Radial Basis Function Neural Network and the mean square error value of MLP is 0.066666 and mean square error value of RBF is 0.0835026 so from the simulation results it is concluded that the Multilayer Perceptron is slightly better than Radial Basis Function. In this paper, the author predicts the idle slots of the channel, the past information of the channel is based on the sensing history and then allow Cognitive radio to select best slot for data transmission. Neural Network Levenberg-Marquardt (NNLM) algorithm is used for to predict the idle slots of a channel. As compare to Multilayer Perceptron (MLP) and Hidden Markov Model (HMM) Neural Network Levenberg-Marquardt (NNLM) algorithm gives fast and accurate prediction results [6]. In [7], for the channel status prediction the author used Contextual sigma-if neural network algorithm for Cognitive Radio and from the simulation results it is observed that the Contextual Sigma-if algorithm improved the accuracy of channel status prediction than Multilayer Perceptron Neural Network (MLP). Contextual Sigma-if neural network algorithm reduces the sensing time for prediction.

In [8] the basic goal of this paper is to utilize the unused spectrum and the author used tv white spaces, UHF \& VHF band is used for spectrum prediction which are not 
currently used by the primary users without causing any interference to the licensed user. The interference can be minimizing to the Primary user by a technique which sense the whole spectrum, to determine the availability of spectrum whether it is busy or idle by spectrum sensing, however spectrum sensing consumes larger amount of energy and reduces sensing time of same spectrum again and again, the problem is mitigate by using predictive method. Secondary users sense only those channels which are predict as an unused at a time. Spectrum predictor is achieved by using neural network multilayer perceptron (MLP) and also it improves the spectrum utilization and also reduced sensing energy.

In [9], In this paper the author provides a longest idle time of unused spectrum to the unlicensed user by using machine learning technique. Four supervised machine learning techniques (two from ANN and two from SVM) are used to investigate the prediction of length of the off period of the primary user. Poisson, Interrupted Poisson and Self similar models are used for the traffic prediction. In [10] neural network is used for spectrum sensing for secondary user which increase the decision accuracy in the presence of noise in the environment. Neural network is an intelligent technique which is used to predict the unused spectrum by adjusting real time operating parameters and to understand the environment and the capabilities as per the requirement of the secondary users

In [11], author used the Back-Propagation Artificial Neural Network for the substitution of the Complicated Frequency allocation and also from the simulation results author differentiate the original Frequency allocation with the Back-Propagation Neural Network and also author concluded that the Back-Propagation gave the accurate results and it resolve the problem of complicated frequency allocation which increases QoS and decreases interference

In [12], the author proposed the Artificial Neural Network for the prediction of unused spectrum and then predicted spectrum assigned to the Secondary user which reduces sensing time of whole band again and again and increases Quality of services (QoS) without causing any interference to the primary user Moreover author proposed that the Cognitive radio can be apply in different domain and Cognitive radio is a promising solution of fixed spectrum allocation

In [13], author used ANN model for the prediction of channel capacity whether it is occupied by the primary user or not cause channel capacity is an important parameter to detect the channel status also author designed the three ANN model with different layers and multiple number of neurons and from the simulation results author concluded that the ANN model 3 is slightly better than model 1 and 2

In [14], the author used Improved-Back-Propagation Neural Network for the Prediction of unused Spectrum to mitigate the problems which occurred by using the Conventional Back-Propagation Neural Network. The Back-Propagation Neural Network enhanced by the gradient momentum descent algorithm and the genetic algorithm. For the ImprovedBPNN model author used channel state information for the prediction of spectrum rather than channel power and also from the Simulation results author concluded that the Spectrum predictive accuracy of Improved-Back-Propagation Neural Network is higher than the Conventional Back-Propagation Neural Network.

\section{Methodology}

As static spectrum allocation is being used worldwide, the use of spectrum doesn't change much rapidly. We can analyse spectrum for certain durations in different time slots (Figure 1, 2 and 3). In Figure 2 it can be analysed that there are variations in amplitude between $1.8 \mathrm{GHz}$ and $1.86 \mathrm{GHz}$. While $1.86 \mathrm{GHz}$ onwards the amplitude is constant and these frequencies can be used by secondary user for transmission. We can use this information to train neural network to predict available slots. Instead of 
continuously sensing the spectrum or moving sequentially from one frequency to other, in search of holes, this method will aid in accurate prediction with less resources consumed.

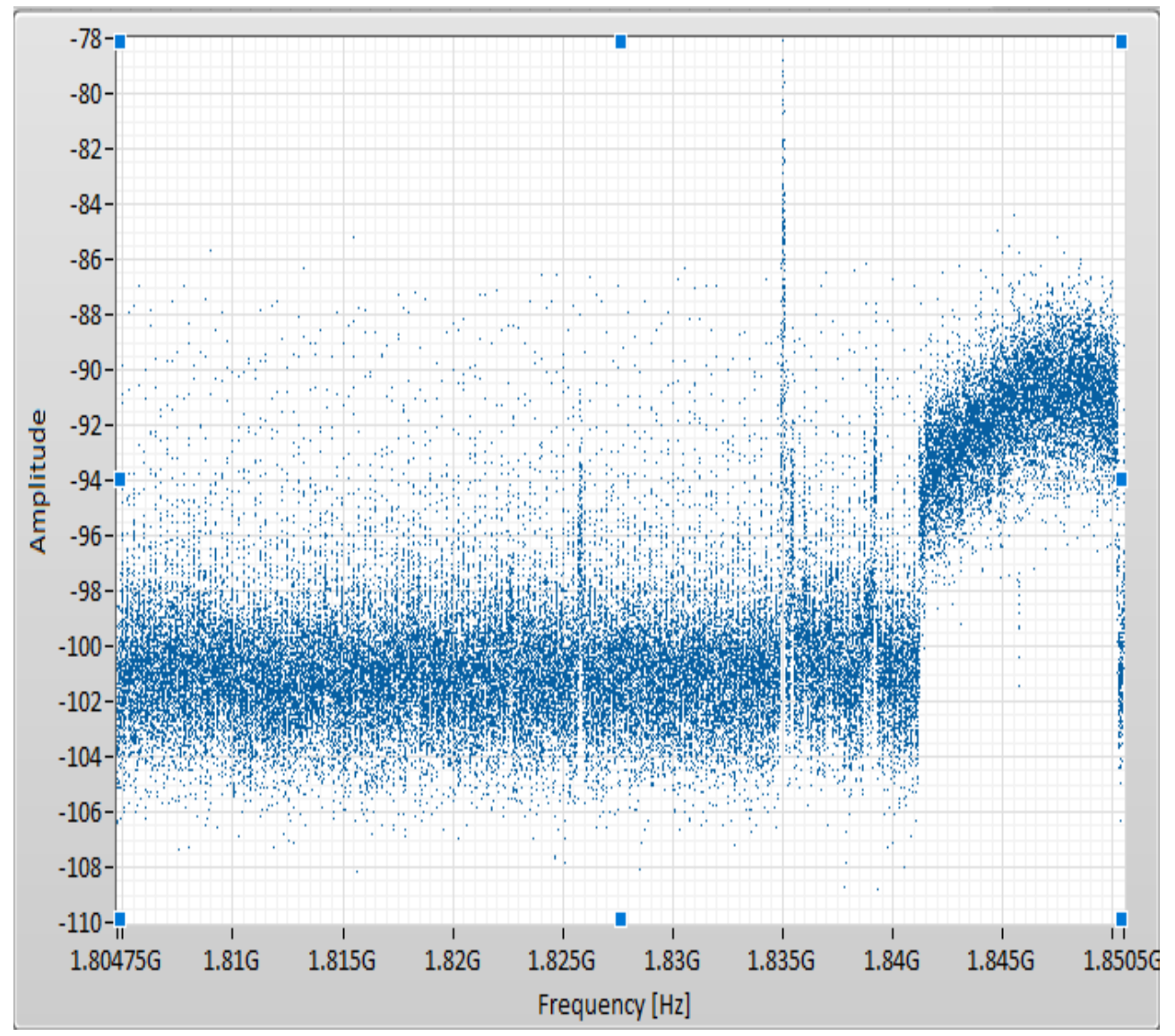

Figure 1. Spectrum Sensing in $1800 \mathrm{MHz}$ Band. Noticeable Change in Amplitude can be Observed

This method will decrease interference. If there is a frequency slot that is usually occupied and remains unused for only a small fraction of time, such slot should not be assigned to secondary user, or avoided until there is no slot available with less occupancy. If spectrum is analysed sequentially for free slots and this slot exists in initial positions, it will greatly impact the performance of the radio. As whenever this slot will be found free, it will be assigned to secondary user and then very soon when primary user will return it will again become occupied and secondary radio, now requires to find a new slot. This search for slot consumes energy and time. It keeps the radio busy in searching for available slot. It in turn also impacts Quality of Service and can cause interference with primary user, multiple time. 


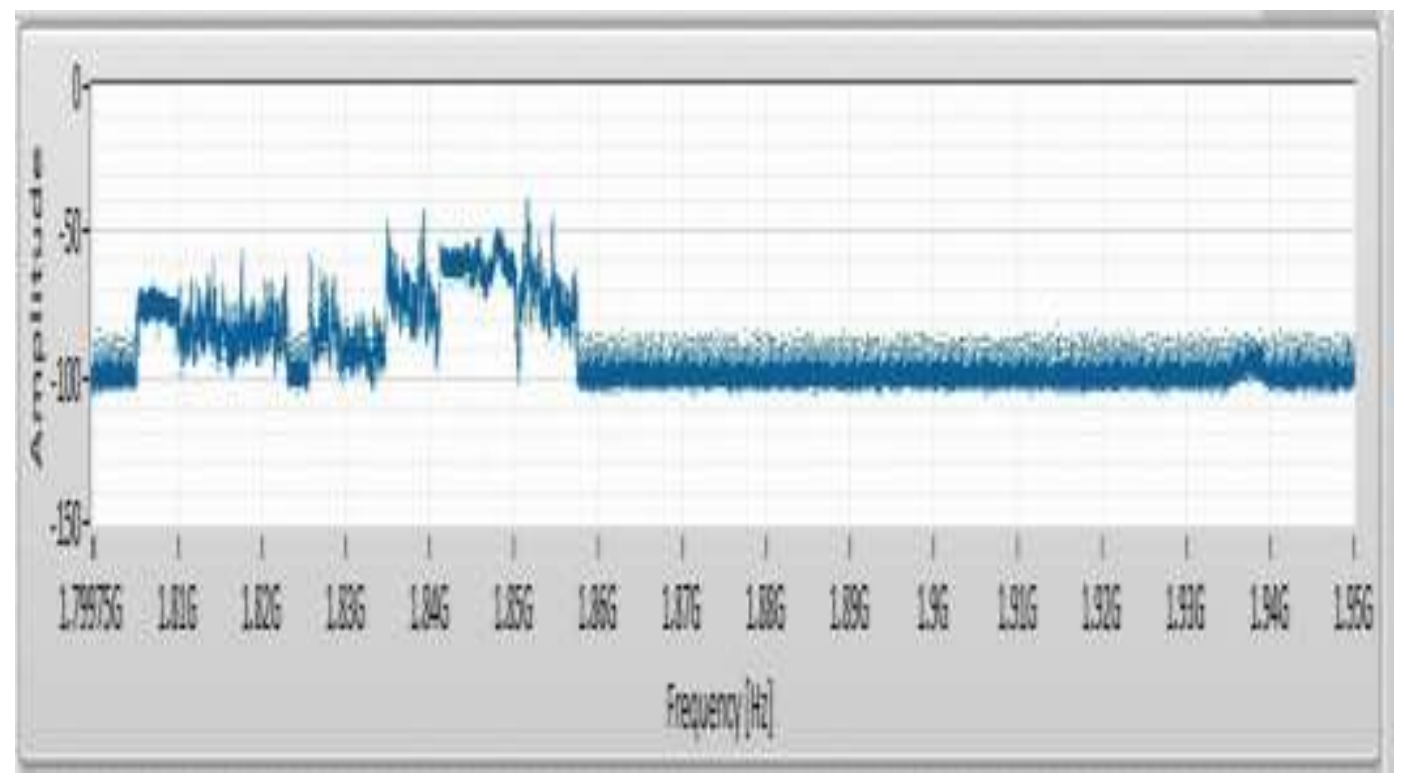

Figure 2. Spectrum Sensing of 1800 and $1900 \mathrm{MHz}$

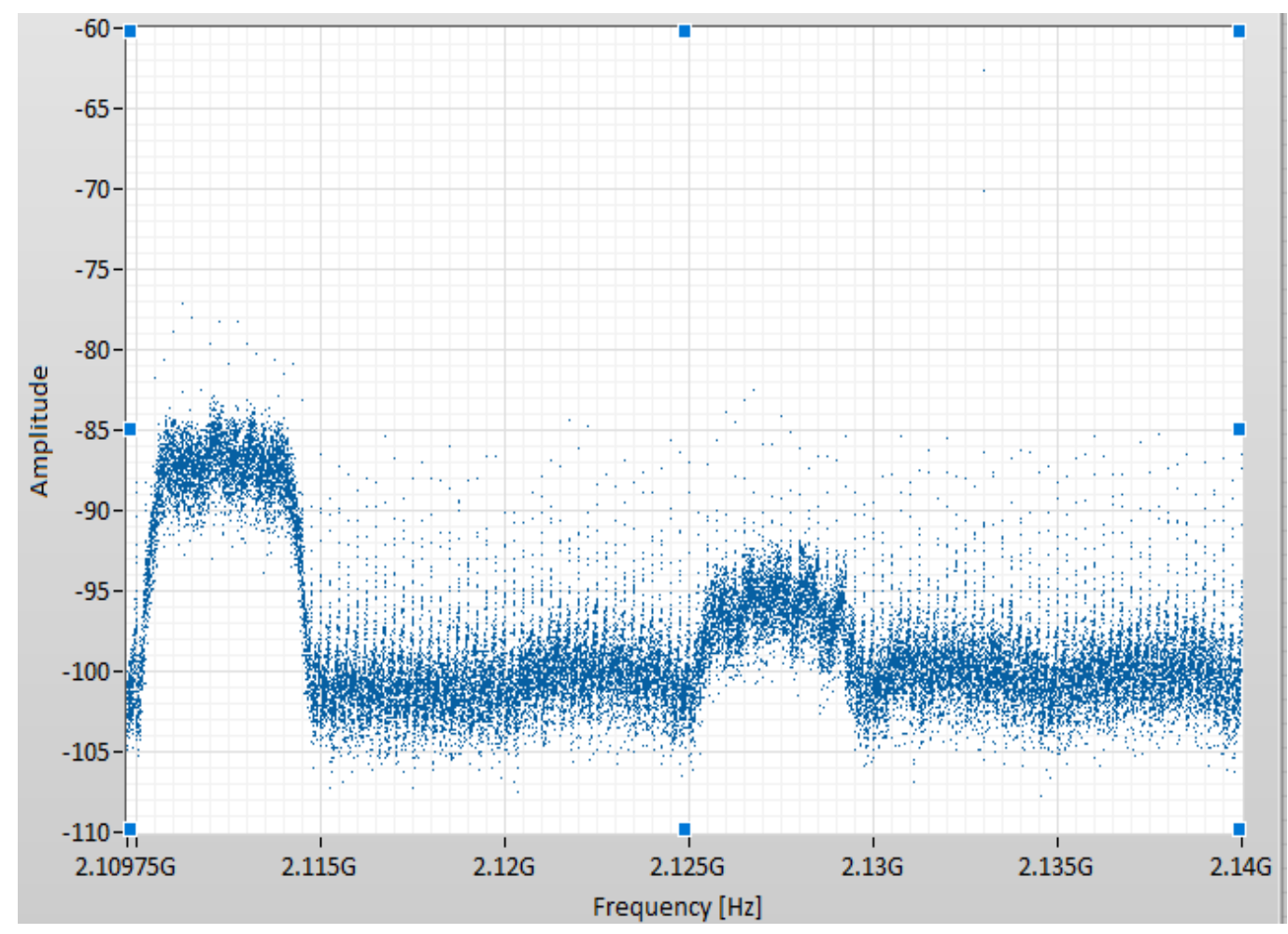

Figure 3. Spectrum Sensing in $2100 \mathrm{MHz}$ Band

We sensed spectrum at different timings in a day. To make dataset more accurate, spectrum sensing is performed three times of each band. We have trained ANN with this dataset. From this dataset ANN can predict slots that are least occupied with primary user. If multiple slots are required, again slots with maximum idle time (least occupancy by primary user) will be selected. These slots will be the slots having maximum idle time among all slots in a particular band predicted using neural networks. 


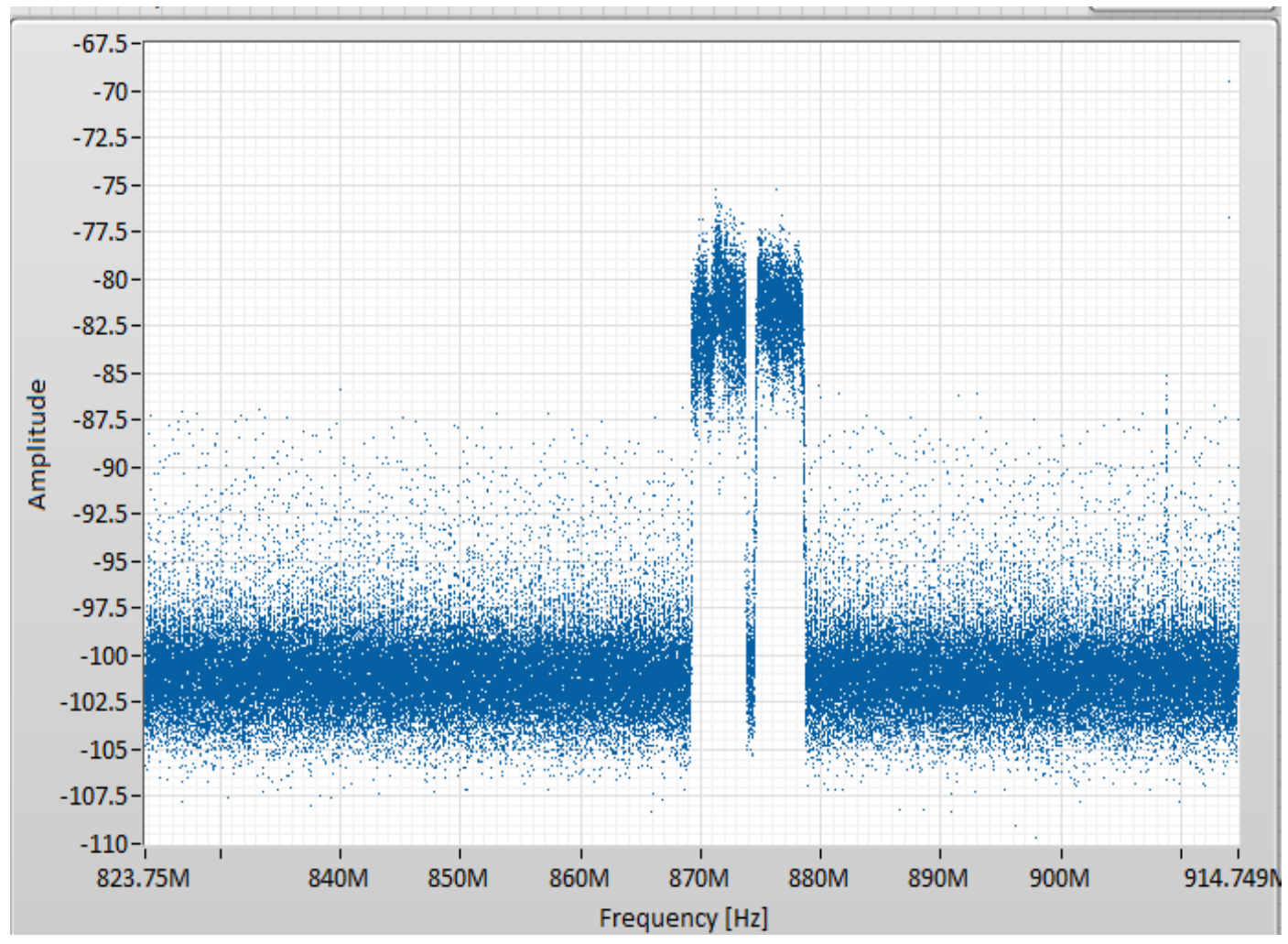

Figure 4. Spectrum Sensing in $824 \mathrm{MHz}$ to $914.9 \mathrm{MHz}$ Band

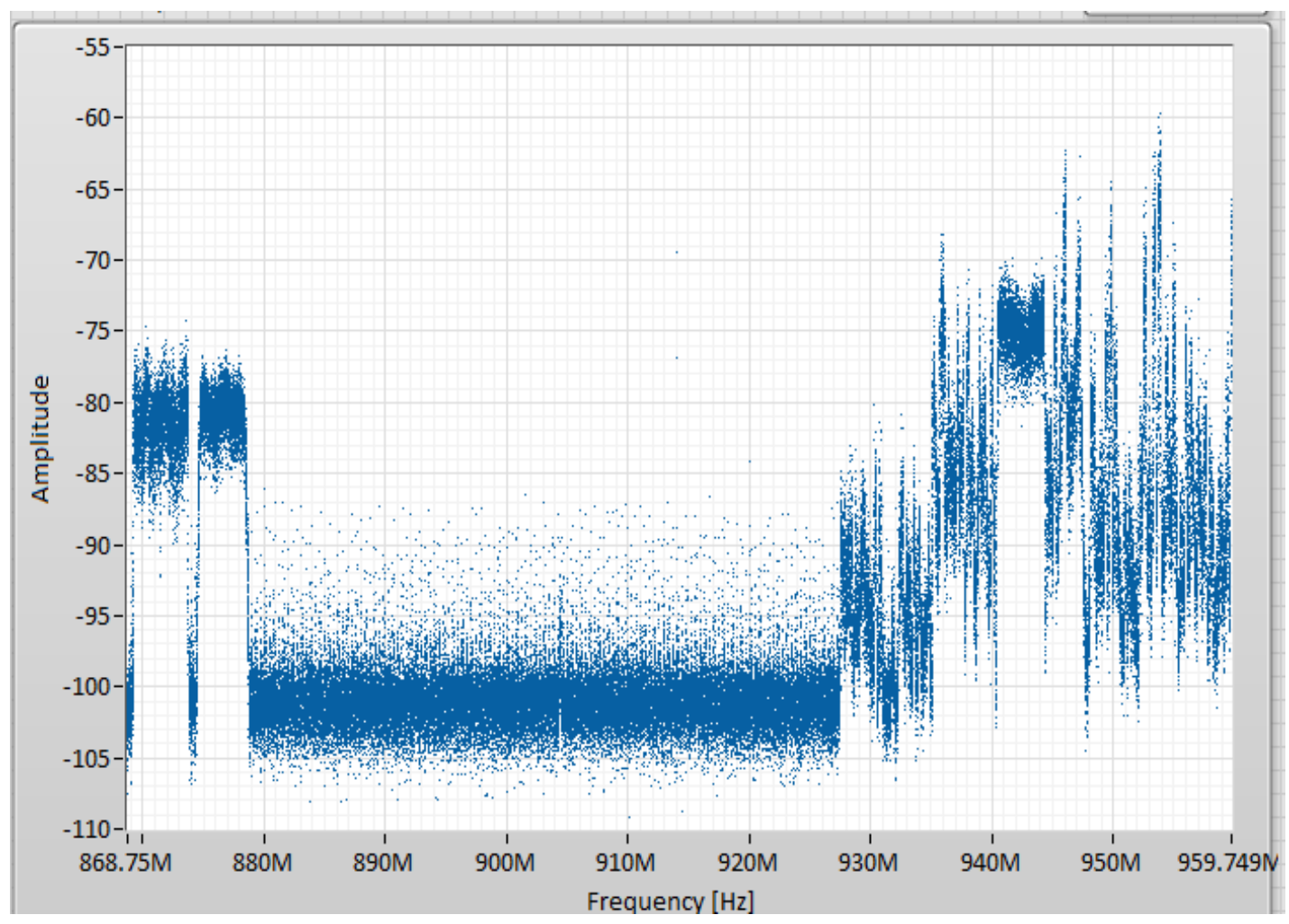

Figure 5. Spectrum Sensing in $869 \mathrm{MHz}$ to $959 \mathrm{MHz}$ Band 


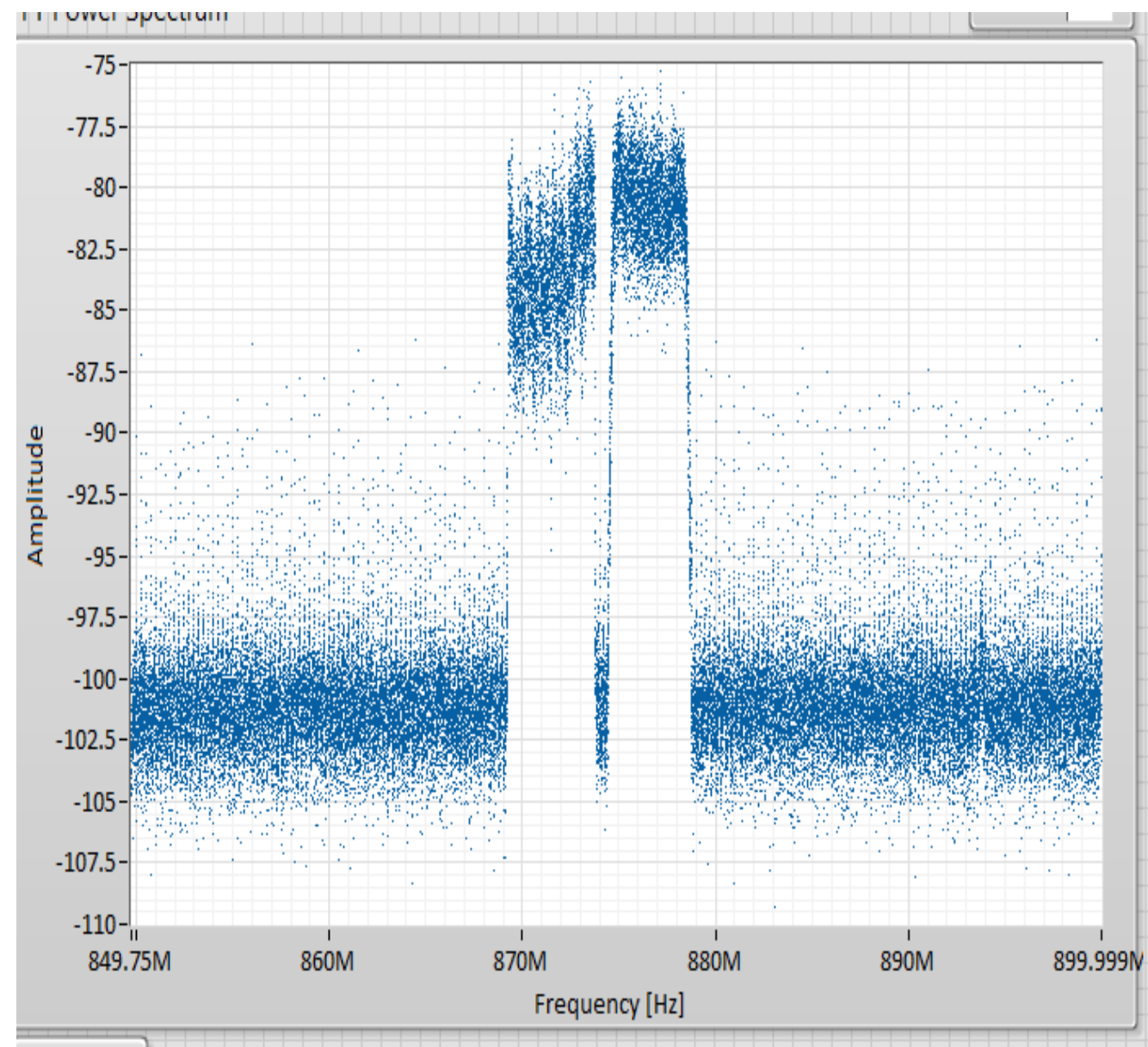

Figure 6. Spectrum Sensing in $850 \mathrm{MHz}$ to $900 \mathrm{MHz}$ Band

When cognitive radio needs a slot, instead of sensing the entire band, it directly reaches the slot which is available and has maximum idle time. This method reduces spectrum sensing time and power consumed for this purpose. As the slot selected is the slot with highest idle, secondary user can continue in that slot for a greater duration which increases QoS and reduces interference. Transmission power of primary user is also known so secondary user will operate at lower frequency. 
Table 1. Spectrum Sensing at Different Bands of Cellular Network

\begin{tabular}{|c|l|r|}
\hline 2 & Frequenc & Amplitude \\
\hline 3 & $2.10975 G$ & -101.27 \\
\hline 4 & $2.10975 G$ & -100.495 \\
\hline 5 & $2.10975 G$ & -103.4 \\
\hline 6 & $2.10975 G$ & -105.869 \\
\hline 7 & $2.10975 G$ & -104.476 \\
\hline 8 & $2.10976 G$ & -103.958 \\
\hline 9 & $2.10976 G$ & -100.949 \\
\hline 10 & $2.10976 G$ & -102.375 \\
\hline 11 & $2.10976 G$ & -101.723 \\
\hline 12 & $2.10976 G$ & -104.733 \\
\hline 13 & $2.10976 G$ & -101.669 \\
\hline 14 & $2.10976 G$ & -100.68 \\
\hline 15 & $2.10976 G$ & -101.175 \\
\hline 16 & $2.10976 G$ & -104.472 \\
\hline 17 & $2.10976 G$ & -100.757 \\
\hline 18 & $2.10977 G$ & -99.3475 \\
\hline 19 & $2.10977 G$ & -100.166 \\
\hline 20 & $2.10977 G$ & -104.772 \\
\hline 21 & $2.10977 G$ & -103.755 \\
\hline 22 & $2.10977 G$ & -102.71 \\
\hline 23 & $2.10977 G$ & -102.026 \\
\hline
\end{tabular}

\begin{tabular}{|l|l|r|}
\hline 23 & $2.10977 G$ & -102.026 \\
\hline 24 & $2.10977 G$ & -99.8115 \\
\hline 25 & $2.10977 G$ & -101.69 \\
\hline 26 & $2.10977 G$ & -104.005 \\
\hline 27 & $2.10977 G$ & -100.429 \\
\hline 28 & $2.10978 G$ & -101.666 \\
\hline 29 & $2.10978 G$ & -102.601 \\
\hline 30 & $2.10978 G$ & -101.025 \\
\hline 31 & $2.10978 G$ & -101.134 \\
\hline 32 & $2.10978 G$ & -102.263 \\
\hline 33 & $2.10978 G$ & -101.109 \\
\hline 34 & $2.10978 G$ & -99.8498 \\
\hline 35 & $2.10978 G$ & -100.687 \\
\hline 36 & $2.10978 G$ & -101.32 \\
\hline 37 & $2.10978 G$ & -101.85 \\
\hline 38 & $2.10979 G$ & -102.893 \\
\hline 39 & $2.10979 G$ & -104.483 \\
\hline 40 & $2.10979 G$ & -102.305 \\
\hline 41 & $2.10979 G$ & -103.189 \\
\hline 42 & $2.10979 G$ & -102.193 \\
\hline 43 & $2.10979 G$ & -99.7906 \\
\hline 44 & $2.10979 G$ & -98.4693 \\
\hline & & \\
\hline
\end{tabular}

\begin{tabular}{|l|l|r|}
\hline 44 & $2.10979 G$ & -102.413 \\
\hline 45 & $2.10979 G$ & -102.5 \\
\hline 46 & $2.10979 G$ & -101.29 \\
\hline 47 & $2.10979 G$ & -99.1416 \\
\hline 48 & $2.10979 G$ & -99.6625 \\
\hline 49 & $2.1098 G$ & -99.3698 \\
\hline 50 & $2.1098 G$ & -99.9279 \\
\hline 51 & $2.1098 G$ & -102.092 \\
\hline 52 & $2.1098 G$ & -101.752 \\
\hline 53 & $2.1098 G$ & -100.065 \\
\hline 54 & $2.1098 G$ & -99.9198 \\
\hline 55 & $2.1098 G$ & -100.534 \\
\hline 56 & $2.1098 G$ & -100.595 \\
\hline 57 & $2.1098 G$ & -99.7034 \\
\hline 58 & $2.1098 G$ & -99.7417 \\
\hline 59 & $2.10981 G$ & -99.6513 \\
\hline 60 & $2.10981 G$ & -101.64 \\
\hline 61 & $2.10981 G$ & -102.481 \\
\hline 62 & $2.10981 G$ & -101.687 \\
\hline 63 & $2.10981 G$ & -103.584 \\
\hline 64 & $2.10981 G$ & -105.799 \\
\hline 65 & $2.10981 G$ & -101.173 \\
\hline
\end{tabular}

\begin{tabular}{|l|l|r|}
\hline 65 & $2.10981 G$ & -101.173 \\
\hline 66 & $2.10981 G$ & -101.2 \\
\hline 67 & $2.10981 G$ & -100.672 \\
\hline 68 & $2.10981 G$ & -100.349 \\
\hline 69 & $2.10982 G$ & -102.019 \\
\hline 70 & $2.10982 G$ & -101.41 \\
\hline 71 & $2.10982 G$ & -101.711 \\
\hline 72 & $2.10982 G$ & -102.078 \\
\hline 73 & $2.10982 G$ & -101.302 \\
\hline 74 & $2.10982 G$ & -99.7117 \\
\hline 75 & $2.10982 G$ & -98.8578 \\
\hline 76 & $2.10982 G$ & -98.3554 \\
\hline 77 & $2.10982 G$ & -103.134 \\
\hline 78 & $2.10982 G$ & -101.014 \\
\hline 79 & $2.10983 G$ & -100.664 \\
\hline 80 & $2.10983 G$ & -102.752 \\
\hline 81 & $2.10983 G$ & -102.24 \\
\hline 82 & $2.10983 G$ & -102.903 \\
\hline 83 & $2.10983 G$ & -101.641 \\
\hline 84 & $2.10983 G$ & -100.369 \\
\hline 85 & $2.10983 G$ & -99.6766 \\
\hline 86 & $2.10983 G$ & -102.031 \\
\hline & & \\
\hline
\end{tabular}




\subsection{Implementing ANN for Smart Cognitive Cellular Network}

Spectrum Scarcity is the main issue in 5G Networks, to resolve this problem, the primary goal of this paper is to improve the spectrum utilization by using Cognitive Radio. Our approach is to Combine Fixed Global Database and locally sensed dynamic database to produce accurate and reliable spectrum database. Using this combination, we can achieve an accurate database that include benefits of both fixed global database and locally sensed dynamic database. For accurate prediction of spectrum holes we require highly accurate database. The database created provide learning and reasoning ability to Artificial Intelligence software that makes fast and accurate predictions without sensing the entire spectrum. As the system is able to make fast predictions for available bandwidth and also increases overall QoS for secondary users. Accurate prediction of spectrum holes/gaps also decrease interference, hence making system Cognitive network reliable. We have used following parameters for the training of dataset

- ANN: Multi layers Perceptron

- Data Division: Random

- Training: Levenberg Marquardt

- Performance: MSE

- Epoch: 1000

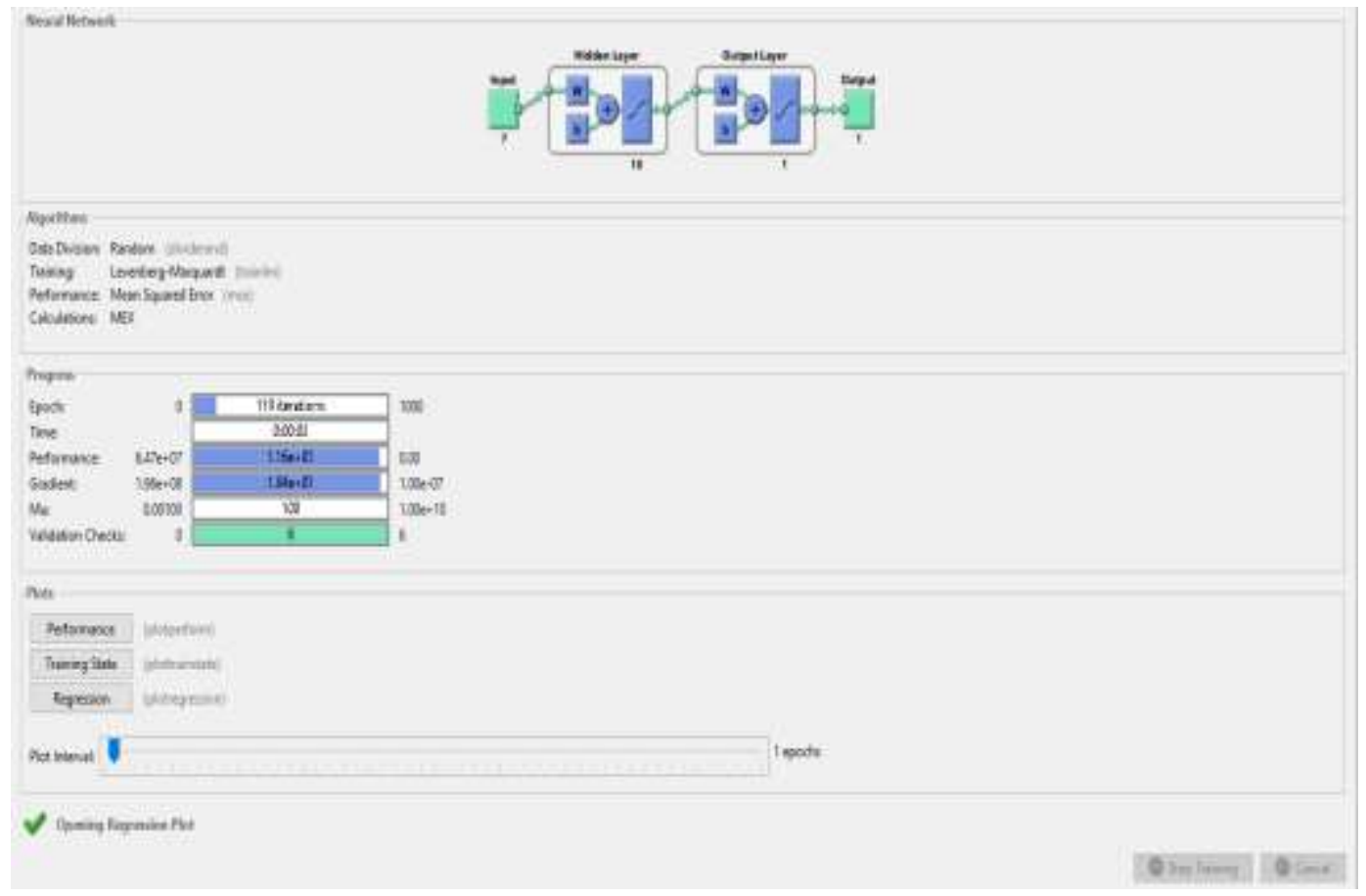

Figure 7. Created Dataset of Cellular Network Import to the ANN 


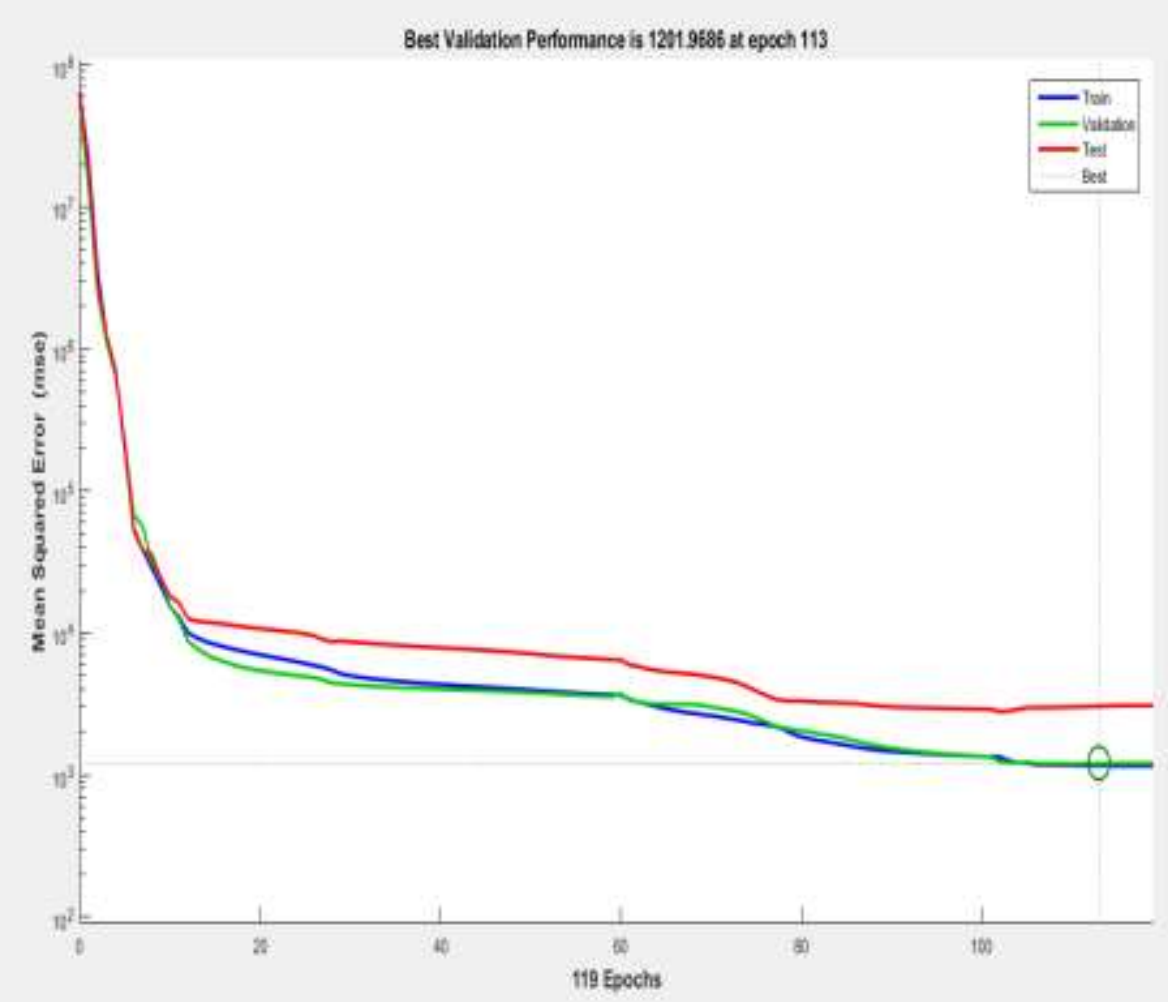

Figure 8. Performance of Trained Created Dataset
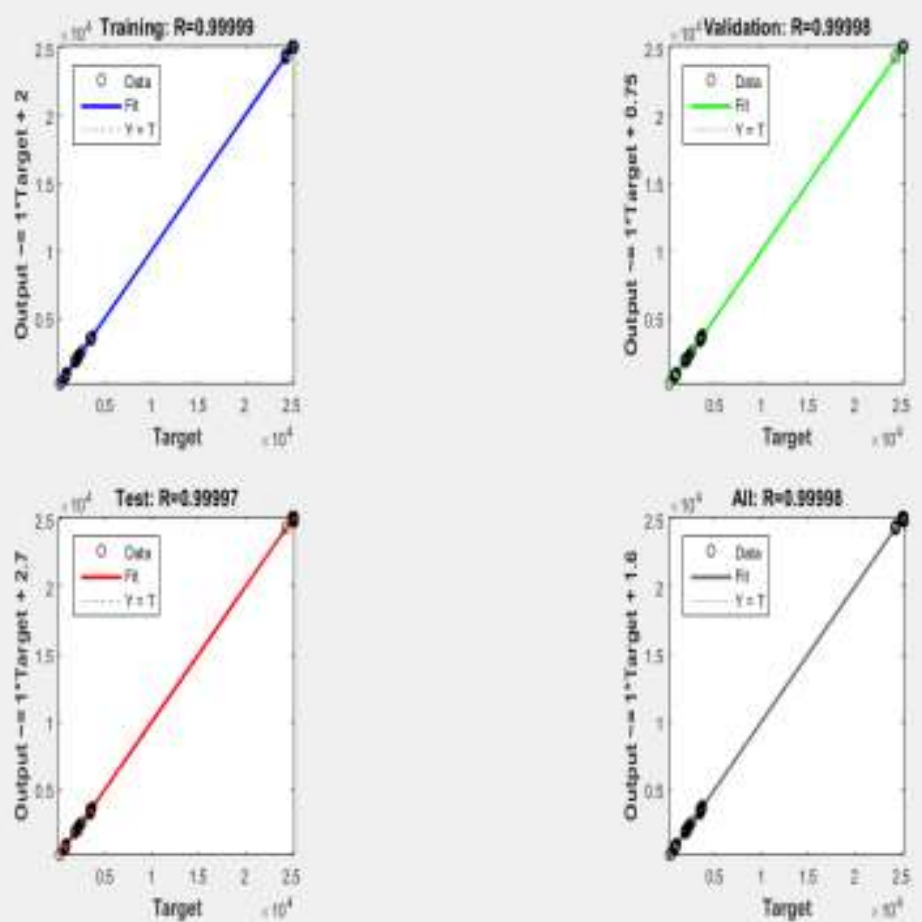

Figure 9. Regression Plot of ANN

\section{Conclusion}

Our proposed methodology uses artificial neural network to solve some major issues in cognitive radio. Using this methodology sensing time and interference between primary 
and secondary user is reduced while QoS and overall system's accuracy increases. Unlike WRANs, this results in better and dynamic frequency allocation and it requires comparatively less resources. Cognitive radio standards require formulation of new laws to allow secondary user to use frequencies owned by primary users. Here we have created dataset of Cellular band and then created dataset is import to the Artificial Neural Network for the prediction of unused spectrum slots. So, we can implement this method in cellular bands as they are greatly affected by spectrum scarcity. Implementation of this method will be more convenient as secondary users will be operating in cellular bands only. Generally idle slots are less likely to occur in Cellular bands and as this method proves to be applicable, hence can be implemented here, it can be implemented easily in most other frequency bands as well.

\section{References}

[1] J. Mitola III and G. Q. Maguire jr., “Cognitive radio: making software radios more personal,” Personal Communications, IEEE, vol. 6, no. 4, (1999), pp. 13-18.

[2] W. Jouini, C. Moy and J. Palicot, "Decision making for cognitive radio equipment: analysis of the first 10 years of exploration”, EURASIP J.Wireless Comm. and Networking, vol. 2012, (2012), p. 26.

[3] V. Krishna Tumuluru, P. Wang* and D. Niyato, "Channel status prediction for cognitive radio network", Wirel. Commun. Mob. Comput., vol. 10, (2010), pp. 1-13.

[4] R. Singh and S. Kansal, "Artificial neural network based spectrum Recognition in cognitive radio", in Conference on Electrical, Electronics and Computer Science, IEEE Students, (2016).

[5] O. Winston, A. Thomas and W. Okello Odongo, "Comparing Performance of MLP and RBF Neural Networks for TV Idle Channel Prediction in Cognitive Radio", Pan African International Conference on Information Science, Computing and Telecommunications, (2013).

[6] P. Roy and Muralidhar, "Channel State Prediction in A Cognitive Radio Network Using Neural Network Levenberg Marquardt Algorithm", in International Journal of Wireless Communications and Networking Technologies, vol. 4, no.2, (2015).

[7] M. J. Mizera-Pietraszko, "Contextual Neural-Network Based Spectrum Prediction for Cognitive Radio".

[8] V. K. Tumuluru, P. Wang* and D. Niyato, "A Neural Network Based Spectrum Prediction Scheme for Cognitive Radio", in the IEEE ICC, (2010).

[9] A. Agarwal1, S. Dubey, M. A. Khan1, R. Gangopadhyay and S. Debnat, "Learning Based Primary User Activity Prediction in Cognitive Radio Networks for Efficient Dynamic Spectrum Access".

[10] R. Singh and S. Kansal, "Performance Evaluation of Neural Network based Spectrum Sensing in Cognitive Radio", 2016 International Conference on Internet of Things and Applications (IOTA) Maharashtra Institute of Technology, Pune, India 22 Jan - 24 Jan, (2016).

[11] X. Tan, H. Huang and L. Ma, "Frequency Allocation with Artificial Neural Networks in Cognitive Radio System", in IEEE 2013 Tencon - Spring.

[12] S. Pattanayak, P. Venkateswaran and R .Nandi, "Artificial Neural Networks for Cognitive Radio", in 2012 IEEE.

[13] S. Pattanayak and R .Nandi, "Identification of Spectrum Holes using ANN Model for Cognitive Radio Applications", in Euro Con, Zagreb, Croatia, (2013).

[14] S. Bai, X. Zhou and F. Xu, "Spectrum Prediction based on Improved-Back-Propagation Neural Networks", in 2015 11th International Conference on Natural Computation (ICNC).

[15] A. Bagwari and G.S. Tomar, "Dual Detectors with Double Threshold for Spectrum Sensing in Cognitive Radio Networks", The Electronics World- The Essentials of Electronics Engineering Magazine, vol.120, issue 1937, (2014), pp.18-23.

[16] A. Bagwari and G.S. Tomar, "Cooperative Spectrum Sensing in MEDs Based CRNs Using Adaptive Double-Threshold scheme", Taylors and Francis - International Journal of Electronics, vol. 101, issue 4, (2014), pp. 37-41. 
International Journal of Future Generation Communication and Networking Vol. 10, No. 12 (2017) 